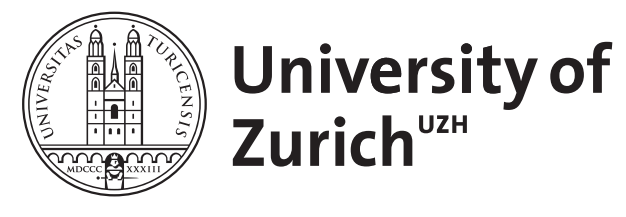

\title{
E-Mail-Kommunikation in Organisationen
}

\author{
Kleinberger, Ulla
}

\begin{abstract}
E-Mails haben in den letzten dreißig Jahren die Kommunikationsnetze in Organisationen grundlegend beeinflusst und weitreichend verändert. Welche Bereiche in welcher Form tangiert sind, wird skizzenhaft in diesem Beitrag ausgeführt. Prägende Textvorlagen für die betriebliche Textausgestaltung müssen überdacht und in einem neuen medialen Kontext gesehen werden. Zusätzlich zu einem Textsortenwandel zeichnen sich inzwischen Grenzen bei der Handhabung der Mails ab: Mails werden teilweise durch die vielfach große Menge an zu bewältigenden Texten als Last wahrgenommen, die täglich bei schwindender Trennung von „Arbeit“ und „Freizeit“ zu bearbeiten ist. Hervorgerufen wird dies durch eine einfach zu nutzende, niedrigschwellige Übertragungsart, die eine Menge an gleichwertig scheinenden, teilweise dialogischen Texteinheiten generiert, die gelesen und verstanden werden müssen. Dies wirft neue Fragen in Bezug auf die sprachliche Identitätskonstruktionen im betrieblichen Alltag, auf dialogische Einheiten, Diskursarten und in Bezug auf Sicherheitsfragen auf.
\end{abstract}

DOI: https://doi.org/10.1515/9783110296235

Posted at the Zurich Open Repository and Archive, University of Zurich

ZORA URL: https://doi.org/10.5167/uzh-169469

Book Section

Published Version

Originally published at:

Kleinberger, Ulla (2018). E-Mail-Kommunikation in Organisationen. In: Habscheid, Stephan; Müller, Andreas; Thörle, Britta; Wilton, Antje. Handbuch Sprache in Organisationen. Berlin: De Gruyter, 167-185.

DOI: https://doi.org/10.1515/9783110296235 


\title{
Ulla Kleinberger
}

\section{E-Mail-Kommunikation in Organisationen}

\begin{abstract}
E-Mails haben in den letzten dreißig Jahren die Kommunikationsnetze in Organisationen grundlegend beeinflusst und weitreichend verändert. Welche Bereiche in welcher Form tangiert sind, wird skizzenhaft in diesem Beitrag ausgeführt. Prägende Textvorlagen für die betriebliche Textausgestaltung müssen überdacht und in einem neuen medialen Kontext gesehen werden. Zusätzlich zu einem Textsortenwandel zeichnen sich inzwischen Grenzen bei der Handhabung der Mails ab: Mails werden teilweise durch die vielfach große Menge an zu bewältigenden Texten als Last wahrgenommen, die täglich bei schwindender Trennung von „Arbeit“ und „Freizeit“ zu bearbeiten ist. Hervorgerufen wird dies durch eine einfach zu nutzende, niedrigschwellige Übertragungsart, die eine Menge an gleichwertig scheinenden, teilweise dialogischen Texteinheiten generiert, die gelesen und verstanden werden müssen. Dies wirft neue Fragen in Bezug auf die sprachliche Identitätskonstruktionen im betrieblichen Alltag, auf dialogische Einheiten, Diskursarten und in Bezug auf Sicherheitsfragen auf.
\end{abstract}

1 Wandel organisationaler Kommunikation durch E-Mails

2 Textsorten, -muster und Praktiken

3 Diskurs und mediale Grenzen

4 Identitätskonstruktion in E-Mails

5 Anwendungsfelder

6 Sicherheit bei E-Mails

7 Literatur

\section{Wandel organisationaler Kommunikation durch E-Mails}

\subsection{Einleitung}

In den letzten drei Dekaden, seit den späten 1980er Jahren, hat sich die Organisationskommunikation durch die Integration von E-Mails/Mails, d. h. von schriftlich verfassten, zeitlich ausgesprochen schnell übermittelten, dennoch asynchronen digitalen Texteinheiten, in weiten Bereichen verändert. Verschiedene Autor(inn)en haben sich dieser Entwicklungen angenommen (als Auswahl einiger Werke, so Beutner 2002; Dürscheid/Frehner 2013; Ziegler/Dürscheid 2002; Jakobs 2003; Frehner 2008; Siegert 2008). Medienwandel sei, so Bucher/Gloning/Lehnen (2010, 10), „stets auch eine Abfolge von unterschiedlichen Medienformaten“. Nachfolgend wird versucht, skizzenhafte Einblicke in ausgewählte Wandelprozesse zu ge- 
ben, die direkt mit Mails in Verbindung gebracht werden können: beispielsweise Einblicke in Textsorten und -musterwandel, in den Wandel von Identitätskonstruktionen und medialen Textnetzen über das einzelne Medium hinaus, bis hin zum Wandel von Anwendungsfeldern und einigen juristischen Aspekten.

Für das Jahr 2013 geht das Technologie-Marktforschungsunternehmen „The Radicati Group“ von 123,9 Milliarden täglich verschickten Geschäfts-Mails weltweit aus. „Statista_1“ versendet von schätzungsweise 3,9 Milliarden Mail-Konten, die Tendenz ist im beruflichen Kontext steigend. Hingegen sind, quantitativ gesehen, private Mails im Rückgang begriffen. Es lässt sich im privaten Bereich dafür eine Verschiebung der gewählten Übertragungsarten hin $\mathrm{zu}$ den social networks (wie Facebook, Linkedin, Xing usw.) beobachten. Ein Großteil der Mails sind Spams oder als nicht-relevante Texte eingeschätzte Einheiten:

Der Anteil der E-Mails, der als unwichtig eingestuft wird, beläuft sich auf $61 \%$ aller versendeten E-Mails. 68,8\% des gesamten E-Mail-Datenverkehrs fällt in die Kategorie Spam. (Libess_1)

Die verbleibenden Mails - immerhin noch über 60 Milliarden täglich - werden aber offenbar als relevant angesehen.

Das konkrete und zeitliche Nutzungsumfeld, in welchem Mails verfasst, gesendet und rezipiert werden, hat sich massiv verändert. Anfänglich wurden Mails eher sporadisch eingesehen, bei weitem war es keine tägliche Routine, wie es heute $\mathrm{zu}$ vielen Berufsprofilen diskussionslos gehört. Noch vor 25 Jahren stieß man auf heftige Ablehnung der digitalen Technik im betrieblichen Alltag. Umfragen in Schweizer Großbetrieben zeigten, dass „die Mehrheit der Betroffenen nur wenig mit den Computern anfangen konnte, beziehungsweise dass sie nur eine sehr geringe Bereitschaft zeigt, Computertechnik in ihre Arbeitsabläufe zu integrieren“ (Kleinberger Günther 2002a, 37, 2003). Zusätzlich waren in den Anfängen die Computer durchwegs stationäre Geräte, sodass man typischerweise sitzend an einem Schreibtisch arbeitete. Heute ist diese Arbeitsweise ergänzt durch eine Vielzahl an tragbaren und mobilen Geräten, die es ermöglichen, örtlich unabhängig im Gehen, im Stehen, sitzend oder liegend seine Mails zu bearbeiten. Das Kommunikationsverhalten und der Effizienzgedanke, der eine Optimierung der zeitnahen - Kommunikation genuin beinhaltet (vgl. z. B. Hahn-Drodofsky 2004), haben sich damit einhergehend verändert: Die Mail-Bearbeitung wurde sozusagen für den beruflichen Alltag „domestiziert“. Weiterentwickelte Applikationen haben unmittelbar Einfluss auf Wahrnehmungs- und Nutzungszufriedenheit, wie Granat (2007) in einer empirischen Studie zur Implementierung von BlackBerry in einem Großunternehmen aufgezeigt hat. Die Erwartungshaltung, schnell auf Nachrichten zu reagieren, ist verstärkt. Parallel dazu steigen die Anforderungen hinsichtlich der Effizienz der Mitarbeitenden (und der Anstieg der geleisteten Arbeitsstunden), der Einfluss auf die permanente Erreichbarkeit selbst im Privatleben. Die Auflösung von Grenzen zwischen „Erwerbsarbeit“ und „Privatleben“ wird hingegen von einem Teil der Befragten als störend erachtet. Die psychische Belastung durch ständige E-Mail-Erreichbarkeit steigt offenbar an. 
Granat (2007) unterscheidet in ihren Überlegungen drei Benutzer-Profile: das „Opfer“, den „indifferenten Nutzer“ und den zufriedenen „heavy user“. Änderungsvorschläge und Weiterentwicklungen werden jeweils auf zwei Ebenen angesetzt: a) Reduktion der Kommunikationsmedien einhergehend mit einem rollenbasierten Managementkonzept („role-based“ Management) und b) einer Änderung der Kommunikationskultur.

Aufgabenbereiche, die über Mails bearbeitet werden, haben sich ausgeweitet. Da es heute der unmarkierte Normalfall ist, mindestens täglich, wenn nicht sogar permanent per Mail erreichbar zu sein, können alle Themenfelder bearbeitet werden - allenfalls eingeschränkt durch Klauseln der Vertraulichkeit, Ansprüche an Geheimhaltung und durch individuelle Schreibkompetenzen. Die „Chronemik des Kommunikationsgeschehens“, wie Dürscheid $(2005,96)$ es formulierte, hat sich grundlegend geändert.

Folgt man McLuhans (1995/1962) Gedankengang, dass sich durch das Implementieren neuer Medien der bestehende Medienfächer anders verteilt, sieht man eine vergleichbare Entwicklung im Umfeld der Mails. Von der traditionellen Briefpost und von weiteren schriftlich genutzten Medien wie Fax, Telex, Telegramm etc. wurden wesentliche Teile übernommen (siehe Kleinberger Günther 2002a); ebenso werden Anteile aus dem mündlichen, face-to-face-Übertragungsbereich, aus Telefongesprächen (Schmitz 2002a) und anderen medialen Übertragungen in den Mails aufgegriffen. Businessmails haben sich etabliert und sind eine feste Größe im betrieblichen Alltag geworden. Wobei inzwischen verschiedene Organisationen weitere kommunikative Tools entwickeln, die sich von der Idee her eher an den social network-Möglichkeiten anlehnen und die in die Jahre gekommenen Mails teilweise ablösen bzw. eine funktionale Änderung verstärken werden. Ein übergreifender, gemeinsamer, internationaler Standard für Mails, der für eine globale Kompatibilität unumgänglich wäre, hat sich bis heute noch nicht durchgesetzt. Nach wie vor sind Mail-Applikationen - so wie andere mediale Übertragungsarten - noch nicht „sicher“, selbst wenn dies von firmeninternen Verschlüsselungstechniken oder Anbietern von secure mail suggeriert wird. Auch in diesen punktuell erwähnten Bereichen wäre noch Koordinationspotential vorhanden.

\subsection{Mailmanagement der Mitarbeiter(innen)}

Die Anforderungen an den Umgang mit den Mail-Daten sind mit den Jahren gestiegen: Lese- und Bearbeitungszeit, Verwaltung und Archivierung, Priorisierung der Arbeiten usw. muss organisiert sein und eingehalten werden, sonst werden die User(innen) vom quantitativen Anstieg der zu bearbeitenden Mails geradezu überrollt. Inzwischen werden einige betriebliche Abläufe langsamer und unproduktiver, da Mitarbeiter(innen) durch die Mail-Menge überfordert sind und in diesem medialen Umfeld nicht ausreichend geübt sind, Strukturen zu implementieren, die 
es ihnen ermöglichen, Prioritäten zu setzen. Ein Mitarbeiter einer Beratungsfirma formulierte es in einem FAZ-Artikel folgendermaßen:

Bünning sagt: „Der eigentliche Effekt, durch E-Mail schnellere Entscheidungen zu fördern, ist weg." Zumal manche Führungskraft am Tag 200 oder gar 300 E-Mails bekommt. Effizientes Arbeiten ist da unmöglich. (FAZ_Höfinghoff 2008)

Für einen produktiveren Umgang mit Mails werden in Organisationen vermehrt Empfehlungen ausgesprochen, wer, wem, was per Mail senden darf, wie man die incoming-Mails handhaben soll und in welchem zeitlichen Rahmen Rückmeldungen zu geben sind. Verschiedene strukturelle Einschränkungen werden von Firmen versuchsweise eingesetzt: Keine firmeninternen Mails mehr am Freitag versenden, alle Mails, die während einer Abwesenheit der Mitarbeiter(innen) eingehen, werden automatisch gelöscht usw. Die Ergebnisse dieser Versuche müssen sich aber erst noch bewähren und sich in einem gesamten organisationalen Ablauf sinnvoll eingliedern lassen. Dies ist abhängig von vielen Faktoren, die außerhalb der eigentlichen Textübertragung per Mail liegen, z. B. in der Betriebsgröße, Funktionen der Mails, Schreibanforderungen usw.

\subsubsection{Verfasser(innen)}

Empfehlungen für den Umgang mit Mails werden häufig formuliert (z. B. Song/ Halsey/Burress 2008), beispielsweise wird den User(inne)n nahegelegt, die Adressatengruppe der Empfänger(innen) zu verkleinern und auf Themen fokussierte Texte/Bilder/Material zu schicken. Nicht mehr „jede/jeder“ soll aufgeführt werden, der oder die allenfalls auch noch Interesse hätten oder - vermeintlich - informiert werden sollten, weder im CC noch im BCC. Mails sollten nicht genutzt werden, um Strukturen, Hierarchien und soziales Netzwerk abzubilden (siehe „Identitätsbildung“ weiter unten). Selbst wenn die Entscheidung nicht einfach fällt, jemanden aus dem Verteiler zu löschen, ist das allemal besser, als unreflektiert zu viele aufzunehmen. Der Effekt, dass Mails von gewissen Absenderadressen nicht mehr oder mit einer gewissen Oberflächlichkeit und Verspätung geöffnet werden, kann so reduziert werden. Nicht alles, was man schreiben könnte, sollte auch versandt werden. Gelassenheit ist als Kriterium nicht zu unterschätzen, um die Mail-Flut allenfalls einzudämmen.

Mails als begleitender Botentext für angehängte Dokumente haben sich vollständig im beruflichen Alltag etabliert. Hängt man einer Mail Dokumente an, delegiert man die Dokumentenablage, -archivierung und -strukturierung den Empfänger(inne)n. Alle müssen sich individuell eine Ordnungsstruktur konstruieren, wie sie mit den Daten umgehen wollen. Dieser Aufwand ist groß und wird nicht von allen Mitarbeiter(inne)n strukturiert vorgenommen. Wie Schmidt (2009, 174) schreibt, basieren viele Ordnungssysteme - er spricht über das Web 2.0 - maßgeb- 
lich auf Praktiken der Zusammenstellung von Nicht-Expert(inn)en: „Geteilte Routinen und Erwartungen bleiben [...] zumeist implizit“, aus Beobachtung anderer entstünden Lösungsmuster. Arbeitszeit wird so, unter Umständen, in das Auffinden und Gestalten von Daten und Datenablagen investiert, die für weitere Tätigkeiten allenfalls fehlt.

Seit technisch die Möglichkeiten von Plattformen als Dokumentenablage geschaffen sind, dienen aus Gründen der technischen Ressourcen (Speicherplatz), der Effizienz und der Gleichstellung der Mitarbeiter(innen) Mails weniger als Botenmedium für Anhänge, sondern übermitteln Links, unter denen die Dokumentationen zentral abgelegt und strukturiert für alle relevanten Mitarbeiter(innen) in gleicher Weise zugänglich sind. Selbstverständlich werden entsprechende $\mathrm{Zu}$ gangsberechtigungen gesteuert.

Begleitende schriftliche Arbeitsanweisungen lassen sich über Aufgabenlisten, die parallel Fortschritte zu illustrieren vermögen, verbreiten. Diese Daten können individuell - also unabhängig von einer Mail - eingesehen werden. Sie dienen in kollaborativen Teams als Illustration der Projektfortschritte.

Die komplexe Aufgabe, Vorgehen und Anweisungen per Mail ausformuliert an die Mitarbeiter(innen) zu senden, wird dadurch etwas abgemildert und ermöglicht neue kommunikative Strukturen, die sich über kollaborative Seiten (groupware, virtuelle Projekträume usw.) in hohem Maße innerbetrieblich ausbilden und ausweiten. Damit einhergehend verlagert sich das „Bring“-Prinzip hin zu einem „Hol“Prinzip: Die Mitarbeiter(innen) müssen sich aktiv um die Informationen/Dokumentationen usw. bemühen, Daten und Dokumentationen werden nicht mehr einfach zugestellt, sondern nur der Link zu den Daten. Damit verbindet sich auch eine neue Wandeltendenz für organisationale Mails hin zu einem „Botenmedium“, interaktiver Austausch in Gruppen wird sich voraussichtlich vermehrt auf die kollaborativen Seiten verlagern.

\subsubsection{Rezipient(inn)en}

Für die Rezipient(inn)enseite ist man von Seiten des Managements inzwischen davon abgerückt, Vorzüge der Mails auszuformulieren. Stattdessen werden mehrheitlich Empfehlungen zur Handhabung der Mails und Reduktion der Mailmenge (e-mail overload) gegeben. Neben dem klassischen Rat, nicht „dauernd“ Mails anzusehen, sondern zeitliche Prioritäten zu setzen und beispielsweise ein-, zwei- oder dreimal täglich seine Mails zu überprüfen, gibt es eine Bandbreite an Ratschlägen (unerwünschte) Post abwehren, Filter nutzen, Metadaten verarbeiten, mehrere hierarchische Priorisierungskategorien einführen, was wann bearbeitet wird usw.; siehe dazu u. a. Kammerer 2013; Mumford 2015).

E-Mails und die damit verbundene „schnelle“ und unkomplizierte Erreichbarkeit sind nicht nur ein Segen, sondern inzwischen offenbar eine Herausforderung 
für die Mitarbeiter(innen) und deren Vorgesetzte. Der - heute zumeist noch ungesteuerte - Erwerb in der Handhabung dieses niedrigschwelligen Tools bringt kollateral unerwünschte Nebenwirkungen mit sich, die in der Zwischenzeit den beeindruckenden Vorteil, den die Mails etabliert haben, überdecken.

\section{Textsorten, -muster und Praktiken}

In den Anfängen der Mails, den 1970er und 1980er Jahren, wurden textkonstituierende Elemente aus dem Umfeld der Programmierer(innen) übernommen. Vieles war möglich, wenig normiert: Ein Pioniergeist ließ sich ausmachen. Immerhin hat man mit den alten, langsamen Modems bei der Einwahl in die Rechner geduldig einige Hürden überwinden müssen, bis man sich in die Mail-Umgebung vorgearbeitet hatte. Mail-Adressen ausfindig machen kam fast detektivischer Arbeit gleich, bis sie sich im deutschsprachigen Raum Anfang bis Mitte der 90er Jahre und im Zuge mit Web $2.0 \mathrm{im}$ 21. Jahrhundert weitreichend etabliert haben. Zwei textkonstituierende normsuggerierende Instanzen, an denen man sich orientierte - oder eben bewusst nicht -, gab es: die Vorlagen durch traditionelle Briefe und die Normen der „Internetfreaks“. Beide Instanzen verfügten über eine Bindungsenergie, die nicht zu unterschätzen war und bis heute noch nachwirkt.

Erst mit der Zeit - und mit der ansteigenden Zahl der User(innen) - entwickelten sich neue Usanzen, etablierten sich neue medienadäquate Konventionen und Modalitäten, gespeist aus unterschiedlichen Bereichen, und bildeten sich bestehende Formen entsprechend weiter (Beispiele dazu in Schnitzer 2012). Inzwischen findet sich eine „Vielfalt an Sprachgebrauchsformen“ (Schmitz 2002b), die den sich neu etablierten Nähe- und Distanzverhältnissen eher entsprechen.

Mails sind eine Übertragungsform einer determinierten Datenmenge, abhängig vom Server und dem Datenanbieter, begrenzt durch voreingestellte Funktionen, durch Programme und Organisation, bestimmte Oberflächenstrukturen (Header, Zeit, Absender, CC/BCC, Betreff und Textkörper usw.), durch unternehmensinterne Richtlinien, der sozialen Usanz in der jeweiligen Organisation und der individuellen Ausprägungen der schreibenden Personen. Diese Konstellation ist speziell und hebt die Mailtexte von anderen organisationalen Texten ab, es bedeutet jedoch nicht, dass die realisierten sprachlichen Phänomene exklusiv und einmalig wären. Die weitaus meisten Elemente finden sich ebenso in anderen Textumgebungen und Übertragungsarten, etwaige Ausnahmen, z. B. Smileys, spezielle Akronyme usw., werden konfrontativ diskutiert, unterschiedlich gewichtet und eingeordnet. Auffallend in Mails ist weniger die Einzigartigkeit der Phänomene als die Ballung existierender Merkmale in bestimmten Konstellationen.

Spezifische Formate, Textsorten und Gattungen ermöglichen es Produzent(inn)en und Rezipient(inn)en, strukturelle Ordnung, Sinn, Aussagegehalt und Wichtigkeit in den Mails effektiv und zeitnah zu konstruieren. Bucher/Gloning/ 
Lehnen $(2010,10)$ bezeichnen dies als „Koordinationsmechanismus“, der die „Kontinuität der Medienkommunikation“ sichert. So wie Ordnungen Stabilität im Umgang zu sichern vermögen, so eröffnen sie den Spielraum für Modifikationen und Variationen. Dabei sind Formate, Textsorten und Muster einerseits Rahmung für Handlungen, andererseits Ergebnis einer Handlung. Medienwandel ist dementsprechend komplex, Bucher/Gloning/Lehnen (2010, 11-13) skizzieren fünf Bereiche, die reflektiert werden sollten: 1) verschiedene (technische) Dimensionen, Rezeptionsweisen und Gattungen, 2) ein „multifaktorieller Bedingungsrahmen“ (zitiert nach Hickethier 2010) unter Zusammenwirkung von „,sozialen, kulturellen, ökonomischen und nutzerbedingten Faktoren“ (Bucher/Gloning/Lehnen 2010, 11), 3) eine unterschiedliche Hierarchisierung von Entwicklungsphasen mit variierender Dynamik, 4) „stratifikatorische Strukturen“ auf Mikro-, Meso- und Makroebene, die sich gegenseitig beeinflussen und bedingen, 5) crossmediale Wandelprozesse. In der Schnittstelle einer systemfunktionalen und wissenssoziologischen Auffassung, so Bucher/Gloning/Lehnen (2010),

erfüllen kommunikative Gattungen die Funktion, die für eine Wissensgesellschaft konstitutiven Kommunikationsprozesse $\mathrm{zu}$ routinisieren und damit überflüssige rationale Aushandlungsprozesse [...] zu vermeiden. (Bucher/Gloning/Lehnen 2010, 25)

Damit ermöglicht man eine Innensicht auf Texte und die konstituierenden Strukturen und Elemente wie auch eine Außensicht der Textvernetzung und diskursiven Umgebung. Beides kann zwar analytisch getrennt angegangen werden, beeinflusst sich jedoch gegenseitig. Um Wandelprozesse, ausgelöst durch Mails, fassen zu können, müssen diese unterschiedlichen Ebenen in ihrer gegenseitigen Abhängigkeit und Beeinflussbarkeit reflektiert werden. Auf analytischer Ebene kann so ein umfassender Zugang geschaffen werden, wenn einzelne Phänomene in einem größeren systematischen Zusammenhang gesehen werden. Beispielsweise ändern sich in concreto nicht nur die Anrede- und Grußfomeln in den Mails, sondern der kommunikative Zusammenhang, in denen sie verwendet werden, sowie die Art der adäquaten Verwendung erleben einen funktionalen Wandel. Mails stehen in einer weitaus umfassenderen Entwicklung, nur in ihnen spiegelt sich die Entwicklung täglich.

Häufig diskutiert werden beispielsweise Fragen der normierten Sprachrichtigkeit, die von Kulturpessimist(inn)en als „Sprachzerfall“ gedeutet, von anderen hingegen als „Wertewandel“ gesehen werden. Es stimmt, dass nicht alle gesendeten Mail-Texte strengen traditionellen Normvorstellungen entsprechen. Hingegen lässt sich ein Zusammenhang zwischen Modalität und Sprachrichtigkeit aufzeigen (siehe $\mathrm{zu}$ den Entwicklungen jugendlicher Schreiber(innen) Kleinberger Günther/ Spiegel 2006). Diese Tendenz ist ebenfalls in beruflichen Kontexten aufzeigbar: Normnähe und Normferne der Texte sind pragmatische Indikatoren für Modalitäten und textliche „Priorisierung“. Flexible Handhabung und Abweichungen von der Norm sowie explizite Normferne sind Indikatoren und eine Graduierung für einen vertrauten, freundschaftlichen Umgang; starke Normierungen und Anleh- 
nung an klassische textliche Normwerte hingegen sind Indikatoren für einen offiziellen Charakter der Texte. Zuordnungen sind relative Werte. Dass die sprachliche Gestaltung der Mail sich jedoch näher am „Mündlichkeitspol“ (Dürscheid 2009, 56) orientiert, kann nicht verallgemeinert werden.

\subsection{Dialogische Struktur}

Mails etablieren in vielen Fällen punktuelle oder längere dialogische diskursive Einheiten (Bittner 2003, 154), in denen aufeinander Bezug genommen wird und die in „Mail-Dialogen“ abgebildet sind (Siever 2006, 76 bezeichnet sie noch als „Quasi-Dialoge“). Dies hat sich in der letzten Dekade durch das Etablieren von adaptierter Software markant geändert und sich - den dialogischen Gegebenheiten und Anforderungen, die aus anderen Textübertragungsprogrammen bekannt sind, so WhatsApp, bei SMS u. a. m. - angepasst.

Individuell versandte, singuläre, „kontextlose“ Mails, auf die nicht geantwortet wird, sind eher selten. Meist sind Bezugnahmen linear von Mail zu Mail im Sinne von Threads nachzuverfolgen, ab und an aber wird auch auf länger zurückliegende Einheiten referiert. Textliche Bezüge zwischen verschiedenen Mails sind eine Selbstverständlichkeit. Falls verschiedene Themen und Funktionen in einer Mail abgearbeitet werden, können deshalb mehrere thematische und inhaltliche Bezüge parallel in derselben Textumgebung realisiert werden. In der Forschung hervorgehoben werden stilistisch eingesetzte Adjazenzellipsen (Dürscheid 2005, 91; Dittmann 2006), die einen möglichst unmittelbar vorangehenden Bezugstext voraussetzen, um sinnvoll und zeitnah aufgelöst werden zu können. Außerhalb von dialogischen Strukturen sind sie schwer verständlich und eröffnen einen großen, vagen Interpretationsraum.

\subsection{Quotes}

„Quotes“, also das (automatische) Zitieren von bestehendem Text, ist den Mails in dialogisch-diskursiven Situationen eigen. Über eine einfache „Reply“- oder „Forward“-Funktion wird Text zitiert, der sich farblich meist von den Vorgängertexten abhebt. Vielfach entstehen über diese Funktion längere Textketten eines Diskurses. In Beutner 2002 wird die Verwendungsweise von „Quotes“ beschrieben, wobei in nur einer von 134 untersuchten Mails automatisch zitiert wurde. Im Allgemeinen wird so eine - vermeintlich verlässliche - Rekonstruktion des kommunikativen Ablaufs und eine Form der Archivierung ermöglicht. Jedoch sind es keine zuverlässigen Quellen. Bei jedem Reply und Forward kann die schreibende Person den Text der Quotes abändern, ergänzen, ersetzen oder löschen, ohne dass es den Empfänger(inne)n direkt und ohne Aufwand ersichtlich wäre. Dürscheid $(2005,96)$ vermutet, dass sich durch die intensive Nutzung der „Quote-Technik“ diskursive Einhei- 
ten mehren, die eine schrittweise Entwicklung einzelner Themen förderten, und sich damit die verwendeten Zeichenzahlen pro Mail reduzierten. Dazu gibt es meines Wissens keine quantitativen Analysen, die diese Vermutung belegen könnten. Festhalten lässt sich hingegen, dass die Quote-Technik extensiv genutzt wird, heute als den Mails inhärent angesehen wird, dialogische Bezüge erleichtert, ohne dass jedoch der Mehrwert dieser Möglichkeiten aufgezeigt worden ist. Allenfalls leistet dieser quantitative Zuwachs an Textmenge ebenfalls einen Beitrag zur individuell wahrgenommenen „Mail-Flut“.

\subsection{Textsorten und -muster}

Sämtliche schriftlichen organisationalen Textsorten und -muster können in Mails vorkommen: Beschreibungen, Vorgehensweisen, Sitzungseinladungen, Protokolle, Offerten, Verträge, Mahnungen, Aushandlungen zu Themenbereichen, sozial relevante Texte wie Geburtsanzeigen, Todesanzeigen, Einladungen, etc.

Normative Textsortenvorstellungen und die Ränder traditionell etablierter Textsorten werden in Mails flexibler gehandhabt. Beobachtbar sind Aneinanderreihungen verschiedener Textsorten in eine Übertragungseinheit, Vermischungen, die Emergenz schaffen und im Einzelfall neue Textsorten entstehen lassen, siehe dazu beispielsweise Gansel (2008). Genuin Neues entsteht ausgesprochen selten. Eher kristallisieren sich Muster heraus, die sich aus vorhandenen weiterentwickeln: neue Standards bei Texteinheiten, adaptierte Textbausteine, Verwendung von Smilies, um Sachverhalte handlungsorientiert zu verdeutlichen (z. B. als Indikator für Ironie). Texte werden medial adaptiert, teilweise bedingt durch die Maske der Mails, z. B. Übernahme der Betreff-Zeile aus den Briefnormen, oder es werden neue Muster entworfen (Anordnungen von Header, Verteiler, Absätze, Einzüge, Signaturen, Layout der Schreiben in Abhängigkeit von der Bildschirmgröße). Aspekte wie Höflichkeit, Nähe und Distanzregelung passen sich den Anforderung der digitalen Übermittlung an, gewählte Einheiten vermögen sich teilweise durchzusetzen, teilweise nicht (siehe auch Thaler 2012; Spiegel/Kleinberger 2011). Neu ist sicherlich die reichhaltige Erfahrung von User(inne)n, inhaltliche Aspekte schriftlich auszuhandeln und manchmal daran zu scheitern. Die Macht der Schriftlichkeit will für bestimmte Situationen erworben und professionalisiert werden. Beispielsweise können schriftliche Anweisungen per Mail übermittelt werden - eine Textsorte mit einer bestimmten Funktion, in der es keine ausgeprägte schriftliche Tradition gab, an die man sich anlehnen konnte. Die feinen sozialen Differenzen schriftlich zum Ausdruck $\mathrm{zu}$ bringen, stilistisch geschickt zu verfassen und für verschiedene Rezipient(inn)en adäquat zu formulieren, ist eine Kompetenz, die nicht „einfach so“ gegeben ist, sondern die mit viel Engagement und Reflexion erworben werden kann.

Die Möglichkeit, Texte beziehungsweise Dateien an einen Text anzuhängen und allenfalls kommentiert zu versenden, macht Mails zu einem multifunktionalen 
Trägerboten. Sie unterliegen keiner semiotischen Beschränkung, sofern die Informationen digitalisierbar sind.

Neben den traditionell schriftlich ausgeprägten Textsorten werden ausgewählte, herkömmlich eher im mündlichen Bereich realisierte Gesprächssorten verschriftlicht, so beispielsweise Terminabsprachen, Arbeitsanweisungen, Informationsverteilung, Elemente aus der Softcommunication (siehe Kleinberger Günther 2005; Kleinberger 2008) etc. Texte selbst wandeln sich, ebenso wie die Art der Realisierung sich den gegenwärtigen Anforderungen und Möglichkeiten anpasst. Fragen der „Persönlichen Öffentlichkeit“, also „Informationen von persönlicher Relevanz mit anderen [auch in einem beruflichen Kontext, Anm. der Verf.] zu teilen“ (Schmidt 2009, 126) stellen sich auch für Mailtexte.

Variationen bestehender Textsorten und -muster sind die Norm. Wright (2013) arbeitet aus einem der wenigen zugänglichen E-Mail-Korpora (Enron in den USA) heraus, dass man von „Genre-lecten“ der einzelnen Schreiber(innen) sprechen kann: Sie verfügen zwar über einen stilistischen Fächer, wählen aber ähnliche sprachliche Realisierungen je nach medialer Umgebung. Gerade für die Forensik ist das ein wichtiger Ansatzpunkt für medial übermittelte Texte.

\section{Diskurs und mediale Grenzen}

„E-Mails bieten mehr technische Möglichkeiten als herkömmliche Briefe“ schreibt Dürscheid (2005, 89), was selbstverständlich zutrifft. Gleichzeitig aber bieten Mails auch weniger Umsetzungsmöglichkeiten als traditionelle Briefe. Ausgestaltungen sind vorerst noch mediale Grenzen gesetzt, Olfaktorisches kann (noch) nicht realisiert werden - die Zeit der mit lieblichem Rosenparfüm besprenkelten büttenpapierenen Briefe ist wohl weitgehend vorbei -, Taktiles ist nicht relevant, eine Graduierung der Wichtigkeit gelingt deshalb über wenige semiotische Symbole und typographische Möglichkeiten, z. B. der Trippelung von Interpunktion. Abgesehen von diesen wenigen gestalterischen Möglichkeiten erscheinen die Mails im incoming-Mailordner in ihrer statischen Auflistung gleichwertig und gleich „wichtig“.

Mit Mails gelingt es dennoch relativ leicht, die genuinen Grenzen des schriftlichen Mediums zu durchbrechen bzw. andere Medienarten integrativ trans- und intermedial aufzunehmen. Das Einfügen eines Links in die Mail ermöglicht mit einem Klick in eine andere Applikation zu wechseln, vom Schriftlichen ins Mündliche, vom lesenden Rezipieren in andere visuelle Darstellungen mit bewegten oder statischen Bildern, Ton usw.

Mails sind eingebunden in komplexere mediale Strukturen, „Mediengrenzen“ werden mehr und mehr nebensächlich, was eine Durchmischung fördert.

Language comes integrated in visually organized environments, verbal exchanges tend to be more fragmented and dependent on multimodal context, and meaning is constructed through the interplay of language and other semiotic means. (Androutsopoulos 2011, 279) 
Grenzen können, bedingt durch die mediale Einbettung der Mails und durch die temporale Unmittelbarkeit des Mediums, umgangen und in einer neuen Konstellation realisiert werden: Beispielsweise im Bereich der Lebens- oder medizinischen Beratung lässt sich die Zeit zwischen (schriftlichen) Anfragen und (mündlichen) Beratungsgesprächen unter Umständen massiv minimieren. Organisationen, wie Krankenhäuser und medizinische Verbände, bieten Mail-Beratungen an. Sofern Anfragen schriftlich erledigt werden können, wird das gemacht. Zusätzliche Videotelefonie, wie z.B. telemedizinische Leistungen, ist als Unterstützung in einem transmedialen Bereich auf dem Vormarsch (NetCare_1).

\subsection{Bewerbungen per E-Mail - eine exemplarische Darstellung}

Bewerbungen gehören zum Alltag von Organisationen. Diese ursprünglich mündlich geprägte Diskurseinheit entwickelte über Jahre einen schriftlichen Standard, der mit großer Akribie und einem massiven Zeitaufwand in den Schulen eintrainiert wird. Das - schriftliche - Bewerbungsdossier, traditionell bestehend aus einem Motivationsschreiben, dem Lebenslauf, Zeugnissen und Referenzen, unterliegt in den letzten Jahren einem hohen Druck zu Innovation und hin zum Mündlichen. Innovativ sind bei großen, global handelnden Organisationen die online-Dossiers, welche Bewerber(innen) in einer Datenbank mit vorgeformten Containern ausfüllen sollen. Üblicherweise besteht hier die Möglichkeit, Daten in die Datenbank „hochzuladen“, neben schriftlichen Dokumenten kann eine Video- oder Tondatei hinterlegt werden. Wenn solch eine Datei im Bewerbungsportfolio vorhanden ist, wird sie auch ohne großen Aufwand an eine Mail-Bewerbung angehängt werden können. Zukunftsträchtig für Bewerbungen sind die sich rasant entwickelnden E-Portfolios, die mit ihrer Brückenfunktion zwischen Ausbildung und Beruf neue Bewerbungskonstellationen generieren. Der Zugang zum E-Portfolio über einen Link lässt sich einfach in Bewerbungsmails integrieren (siehe beispielsweise Hofert $2010 \mathrm{zu}$ Online-Bewerbungen).

\section{Identitätskonstruktion in E-Mails}

Die Regulierung des sprachlichen und kommunikationsstilistischen Verhaltens von Mitarbeitern zählt zu den augenscheinlichsten Einflussnahmen auf die soziale Identität von Mitarbeitern in ihren verschiedenen Rollen im Unternehmen. (Habscheid u. a. 2015, 399)

Mails - wie andere Texte in Unternehmen - portieren neben den sachlichen Inhalten sprachlich jeweils soziale Aspekte (siehe dazu Kleinberger Günther/Thimm 2000; Kleinberger 2013). Dies geschieht explizit an der sprachlichen Oberfläche oder implizit über „Mitgemeintes“, über Präsuppositionen, semantisch-pragmatische Implikaturen, konzeptuelle Metaphern, Text(an)ordnungen, Diskursverläufe usw., die von 
den Rezipient(inn)en der Texte wahrgenommen, interpretiert und funktionalisiert werden. Diese Diskurse sind

allgemein als regulierende und regulierte Praktiken (vgl. Keller 2005) bzw. musterhafte kommunikative Handlungen [...] zu verstehen, die mittels Zeichenressourcen [...] Bedeutung von sozialer Realität konstruieren. (Meier/Sommer 2012, 98 f.)

In den Mails findet sich eine Besonderheit: Parallel $\mathrm{zu}$ beruflich relevanten Fragestellungen und Themen werden explizit private Ergänzungen ausgeführt. Diese Augenfälligkeit in diesen medial übermittelten Texte kann der Softcommuniation (Kleinberger 2008) zugeordnet werden. Nicht oder nur teilweise strukturierte beruflich relevante Kommunikation, die außerhalb eines zeitlich organisierten Rahmens, wie beispielsweise Sitzungen, spontan stattfindet, wird sowohl mündlich als auch schriftlich realisiert, wobei Mails einen gewichtigen Beitrag leisten, in diesem traditionell eher mündlich übermittelten Bereich, in dem Privates und Berufliches munter gemischt werden können.

\subsection{Normnähe und Normferne - Stil als Indikator}

Das Verfassen von Mails unterliegt verschiedenen Normvorstellungen. Einerseits sollte eine beruflich adäquate stilistische Realisierung umgesetzt werden, andererseits wird eine individuell freundliche Schreibung erwartet (vgl. Meder 2006 mit einem Fokus auf Individualität und Konvention). Der Spielraum ist vergleichsweise groß, Normvorstellungen sind aufgrund der noch jungen Entwicklung der Mails flexibel, koexistierende Formen sind weitgehend toleriert, auch wenn sich „Ankerpunkte“ ausmachen lassen, die als Orientierungshilfe dienen (siehe Androutsopoulos 2011, 284). Inzwischen etablierte Normen, Muster und Praktiken werden durchaus flexibel reproduziert, sodass Abweichungen und Variationen für den Erhalt dieser Dynamik wichtig sind. Anhand von verschiedenen Korpora lassen sich zwei Tendenzen festmachen: eine freundlich unterhaltsame Art, die mit Sprachspiel belustigende Situationen schafft (siehe Felder 2002), oder pointiert ernste und emotional-streitsüchtige Ausprägungen, die eine Heftigkeit in den Raum stellen, die in den analysierten Korpora erstaunlich scheint.

Stil und Stilindikatoren sind Gradmesser für die Konstruktion von Nähe oder Distanz. Bewegt man sich mit Mails in einer konfliktären Situation, brechen die schriftlichen Repliken vielfach ab. Der Konflikt wird offenbar in anderer kommunikativer Umgebung ausgetragen oder sistiert. Diese Beobachtung wird in vergleichbarer Weise in anderen medialen Übertragungsarten gemacht (z. B. Luginbühl 2003; Westera 2007; Empfehlungen zur Vermeidung in Shipley/Schwalbe 2008).

In Texten, in denen unsichtbare soziale Grenzen überschritten, Konflikte ausgetragen oder Vorstellungen wie beispielsweise Höflichkeitsnormen realisiert werden (siehe dazu Kleinberger Günther 2002b), zeigt sich der flexible und wenig normierte Bereich deutlich. 
Web 2.0 site gives rise to social and ideological contrasts, which, depending on the linguistic resources they are given, can find heteroglossic expression. (Androutsopoulos 2011: 294)

Zwar bezieht sich diese Aussage auf Web 2.0, ist aber auch für E-Mails zutreffend. Schriftliche Konfliktaustragungen werden heute in der Berufsbildung (noch) nicht als Kompetenz erworben, eher werden Deeskalationsstrategien im Schriftlichen thematisiert, wenn auch unter Aspekten der „Höflichkeit“.

Allmählich „erobern“ Mails eine gesellschaftliche Anerkennung und Normalität. Sichtbar wird dies nach und nach in der Integration von Texteinheiten. Nicht jeder Text, jede Textsorte wurde über Mail verbreitet, sichtbar wird dies selbst in der Gegenwart beispielsweise bei Todesanzeigen und Kondolenzschreiben. Nach wie vor ist es gesellschaftlich-organisational nicht Usus, Mitarbeiter(innen) per Mail beispielsweise über Todesfälle zu informieren oder zu kondolieren (siehe dazu beispielsweise Gansterer 2008).

\section{Anwendungsfelder}

Mails bestimmen den beruflichen Alltag mit. Fragen, wie man diesen Kommunikationsbereich optimiert, stellen sich aus verschiedenen Fachrichtungen. Wichtig erscheint mir aus linguistischer Sicht, dass neben der Übermittlung beruflich relevanter Informationen Identitäten konstruiert und soziale Beziehungen über schriftliche Texte, Dialoge und Diskurse festgemacht werden. Dies sind iterative Prozesse, die auf bestehende Texte, Textmuster und Praktiken zurückwirken, sie beeinflussen, ändern, neu ausgestalten. Mitarbeiter(innen) werden in diesen Entwicklungen heutzutage jedoch noch nicht geschult. Beispielsweise wird in der Berufsausbildung nicht eintrainiert, wie man kollaborative Schreibprozesse sinnvoll bewältigt, Mails sprachlich effizient nutzt, wie man optimal in dialogischen schriftlichen Situation argumentiert bzw. „streitet“ oder wie interkulturelle Unterschiede oder Missverständnisse am besten gehandhabt und gelöst werden können.

\subsection{Fachkommunikation}

Einen durch Mails ausgelösten Wandel lässt sich im Bereich der Fachkommunikation und der Expert(inn)en-Lai(inn)en-Kommunikation feststellen. Einige vorliegende empirische Untersuchungen basieren auf akademischen Korpora, nur wenige sind aus anderen beruflich relevanten Bereichen.

Der von vielen als eher informell empfundene Status von E-Mails und die leichtere Erreichbarkeit von Fachleuten machen E-Mail allen Risiken zum Trotz zu einem immer wichtiger werdenden Instrument einer dadurch auch mengenmäßig zunehmenden Experten-Laien-Kommunikation. [...] die das Experten-Laien-Verhältnis belasten kann [...]. (Janich 2005, 206) 
Ob und wie diese Verhältnisse durch Mails belastet bzw. entlastet werden, muss differenzierter in einem größeren Kontext untersucht werden. Allgemein ändern sich die Ansprüche, die an die kommunikative Kompetenz von Expert(inn)en und Lai(inn)en gestellt werden - unabhängig von der medialen Übertragungsart.

\subsection{Ratgeberliteratur/Troubleshooter}

Schon kurz nachdem sich E-Mails als Übermittlungsmedium etabliert hatten, wurden Schreibvorlagen, Ratgeber und Troubleshooter publiziert. In den Anfängen meist reduziert auf die „zehn goldenen Regeln“, ergänzt von den zwölf Geboten bis hin zu Netz-Knigge und Dummies-Erläuterungen (so beispielsweise Mandel/ Van der Leun 1997; englisch Lamb/Peek 1995 und deutsch Lamb/Wobst/Peek 1996; Damaschke/Janzen 1998; Damaschke 2005; Erläuterungen für Frauen, Frauenumweltnetz 1995). Sinnvollerweise sind vielfältige Mail-Empfehlungen eingebettet in allgemeine Internet-Ratgeber (z. B. Fraase 1995; Harris/Kidder 1995; BargielaChiappini/Harris 1996). Aufgenommen werden viele denkbare, mehr oder weniger relevante Aspekte: Verteilerlisten, Massenmailing, Werbung, PR, Marketing, Phishing (z. B. Brandt 2009), ebenso wie sprachliche Empfehlungen hinsichtlich Orthographie, Interpunktion, Stil usw.

Speziell thematisiert werden die anwachsenden Fragestellungen rund ums sinnvolle und effiziente Archivieren der Mails (Kupper 2008; Meier 2014; Brossi/ Winkler 2008; Chejnová 2014; Economidou-Kogetsidis 2011; Liu/Dall’Alba 2012; Merrison u. a. 2012; Möllering/Levy 2012; Pasfield-Neofitou 2012a, b; Stroinska/Cecchetto 2012).

\subsection{Interkulturelle Kommunikation}

Mail-Kontakte verbinden lokale, regionale und globale Kulturräume. Eine „verbindende“ Sprach- und Stilform zu finden und zu etablieren, ist eine herkulinische Aufgabe. Englisch als lingua franca hat sich in der westlichen Welt und bei globalen Kontakten deutlich als Verkehrssprache etabliert, Incelli (2013) nimmt beispielsweise Fragen des BELF (Business Englisch as lingua franca) zwischen englisch und italienisch schreibenden Partner(inne)n auf. Die Auswahl an Literatur zu diesem Bereich ist weitläufig, vielfältig und global. Vom geographisch determinierten MailAustausch, z. B. Japanisch-Deutsch von Harting (2011), Englisch-Tschechisch von Chejnová (2014), bis hin zu strukturellen Problemen wird intensiv an Verständnis und Optimierung gearbeitet (z. B. pragmatische Schwierigkeiten von EconomidouKogetsidis 2011; Arbeitsorganisation per Mail zwischen englischen und australischen Studierenden von Merrison u. a. 2012; „facework“ von Stroinska/Cecchetto 2012). 


\section{Sicherheit bei E-Mails}

Erstaunlich ist das verbreitete indifferente Verhalten vieler Mailer(innen) hinsichtlich Sicherheitsfragen beim Versenden von E-Mails (Schwenk 2005). Offenbar scheint das Bewusstsein, dass Mails nicht in einem geschützten Rahmen „sicher“ von A nach B transportiert werden, sondern für geübte IT-Expert(inn)en ohne großen Aufwand eingesehen werden können, nur wenig ausgeprägt zu sein. Selbst in größeren, globalen Unternehmungen werden Maildaten nur in speziellen Zusammenhängen speziell geschützt bzw. verschlüsselt übermittelt - wenn denn überhaupt solche Sicherheitsvorkehrungen genutzt werden. Durch das Setzen verschiedener hierarchischer Ebenen an Berechtigungen ist es jeweils technisch möglich, Mails einzusehen, selbst wenn es ethisch nicht vertretbar ist. Es ist deshalb erstaunlich, wie offen, unkritisch und deutlich, bisweilen verfänglich, manche Texte verfasst sind. Dabei wären Verschlüsselungsmöglichkeiten mit geringem Aufwand zugänglich: Beispielsweise mit PGP (,pretty good privacy“) bzw. GnuPG, um zwei Möglichkeiten herauszugreifen, die öffentlich zugänglich sind.

Je nach nationalen Standards wird der Mail-Verkehr rechtlich geregelt. Das Telekommunikationsgesetz, $\S 110$, erfasst beispielsweise in Deutschland die Maßnahmen zur Überwachung des Mail-Verkehrs. In der Schweiz wird im Artikel 17 des Bundesgesetztes vom 6. Oktober 2000 die Überwachung des Post- und Fernmeldeverkehrs (BÜPF) geregelt und in der Verordnung 780.11 ,über die Überwachung des Post- und Fernmeldeverkehrs“ ausformuliert. In Österreich wurde 2003 das Telekommunikationsgesetzt mit den Angaben zur den Mails (TKG_AT) ergänzt. Neueste Entwicklungen in einem globalen Kontext, z. B. bei Skandalen rund um den US-Geheimdienst NSA, E-Mails von Hillary Clinton im Rahmen des US-amerikanischen Außenministeriums verdeutlichen die Wichtigkeit von Übertragungssicherheit im Internet und im Mail-Verkehr.

\section{Quellenverzeichnis}

\section{Literatur}

Androutsopoulos, Janis (2011): From variation to heteroglossia in the study of computer-mediated discourse. In: Crispin Thurlow/Kristine Mroczek (Hg.): Digital Discourse. Language in the New Media. Oxford, 277-298.

Bargiela-Chiappini, Francesca/Sandra J. Harris (1996): Requests and status in business correspondence. In: Journal of Pragmatics 28, 635-662.

Beutner, Yvonne (2002): E-Mail-Kommunikation: eine Analyse. Stuttgart.

Bittner, Johannes (2003): Digitalität, Sprache, Kommunikation: Eine Untersuchung zur Medialität von digitalen Kommunikationsformen und Textsorten und deren varietätenlinguistischer Modellierung. Berlin (Philologische Studien und Quellen, 178).

Brandt, Astrid (2009): Zur Strafbarkeit des Phishing Gesetzgebung vs. Technologie. Hamburg. 
Brossi, Pietro/Maria Winkler (2008): E-Mail-Archivierung. Leitfaden für die Umsetzung - mit rechtlichen Grundlagen für die Schweiz, für Deutschland und Österreich. Rheinfelden/ Schweiz: BPX-Edition.

Bucher, Hans-Jürgen/Thomas Gloning/Katrin Lehnen (2010): Medienformate: Ausdifferenzierung und Konvergenz - zum Zusammenhang von Medienwandel und Formatwandel. In: Hans-Jürgen Bucher/Thomas Gloning/Katrin Lehnen (Hg.): Neue Medien - neue Formate. Ausdifferenzierung und Konvergenz in der Medienkommunikation. Frankfurt a. M./New York (Interaktiva. Schriftenreihe des Zentrums für Medien und Interaktivität ZMI, Gießen, 10), 9-38.

Chejnová, Pavla (2014): Expressing politeness in the institutional e-mail communications of university students in the Czech Republic. In: Journal of Pragmatics 60, 175-192.

Damaschke, Giesbert (2005): Start mit E-Mail: So geht die Post ab! München.

Damaschke, Gisebert/Birte Janzen (1998): So funktioniert E-Mail: Ein visueller Streifzug durch die elektronische Post. München.

Dittmann, Jürgen (2006): Konzeptionelle Mündlichkeit in E-Mail und SMS. In: Ulrike Reeg (Hg.): Interkultureller Fremdsprachenunterricht: Grundlagen und Perspektiven. 5. Jahrestagung der DeutschlektorInnen an italienischen Universitäten. 23.-27. September 2004, Monopoli (Bari). Bari, 79-97.

Dürscheid, Christa/Carmen Frehner (2013): E-Mail Communication. In: Susan Herring/Dieter Stein/ Tuija Virtanen(Hg.): Pragmatics of Computer-Mediated Communication. Berlin: Mouton de Gruyter (= Handbook of Pragmatics 9), 35-54.

Dürscheid, Christa (2005): E-Mail - verändert sie das Schreiben? In: Torsten Siever/Peter Schlobinski/Jens Runkehl (Hg.): Websprache.net. Sprache und Kommunikation im Internet. Berlin/New York (Linguistik. Impulse \& Tendenzen, 10), 85-97.

Dürscheid, Christa (2009): E-Mail: eine neue Kommunikationsform? In: Sandro M. Moraldo (Hg.): Internet.kom. Neue Sprach- und Kommunikationsformen im WorldWideWeb. Band 1: Kommunikationsplattformen. Rom, 39-70.

Economidou-Kogetsidis, Maria (2011): „Please answer me as soon as possible“. Pragmatic failure in non-native speakers' e-mail requests to faculty. In: Journal of Pragmatics 43/13, 3193-3215.

Felder, Ekkehard (2002): „Der Zwang zur Zwanglosigkeit!“ - Stilistischer Spagat zwischen Konventionalität und Originalität in E-Mails. In: Arne Ziegler/Christa Dürscheid (Hg.): Kommunikationsform E-Mail. Tübingen (Textsorten, 7), 169-185.

Fraase, Michael (1995): The Mac Internet Tour Guide: Cruising the Internet the Easy Way. 2nd ed. Chapel Hill, NC.

Frauenumweltnetz (1995): Computervernetzung für Frauen Mailboxen, Internet und alles andere ein Handbuch für Einsteigerinnen. Zürich/Bern.

Frehner, Carmen (2008): E-Mail - SMS - MMS: The Linguistik Creativity of Asynchronous Discourse in the New Media Age. Bern.

Gansel, Christina (2008): Systemtheoretische Perspektiven auf Textsorten. Vorbemerkungen. In: Christina Gansel (Hg.): Textsorten und Systemtheorie. Göttingen, 7-18.

Gansterer, Helmut A. (2008): Darf man per E-Mail kondolieren? Überleben im Alltag. München. Granat, Judith (2007): The Domestication of Mobile Email in the Corporate Environment and its Strategic Implication for the Next Generation of Communication Applications. St. Gallen: Dissertation.

Habscheid, Stephan/Andreas P. Müller/Britta Thörle/Antje Wilton (2015): Sprache in Organisationen. In: Ekkehard Felder/Andreas Gardt (Hg.): Handbuch Sprache und Wissen. Berlin/ Boston, 392-410.

Hahn-Drodofsky, Roswitha (2004): E-Mail im Organisationsprozess. Renningen.

Harris, Stuart/Gayle Kidder (1995): Netscape quick tour for Macintosh accessing \& navigating the Internet's world wide web. Chapel Hill, NC. 
Harting, Axel (2011): Bitten in E-Mails japanischer Deutschlernender: Schwierigkeiten beim Erwerb zielsprachlicher Textnormen unter Berücksichtigung der Einflüsse muttersprachlicher deutscher und japanischer Textmuster. Baltmannsweiler.

Hickethier, Knut (2010): Einführung in die Medienwissenschaft. 2. aktualisierte und überarb. Aufl. Stuttgart.

Hofert, Svenja (2010): Stellensuche und Bewerung im Internet: Die perfekte E-Mail-Bewerbung. Bewerbung über Online-Formulare. Wichtige Stellenmärkte und Karriereportale. 6. aktualisierte Aufl. Hannover (Beruf und Karriere).

Incelli, Ersilia (2013): Managing discourse in intercultural business email interactions: a case study of a British and Italian business transaction. In: Journal of Multilingual and Multicultural Development 34/6, 515-532.

Jakobs, Eva-Maria (2003): Medien der Individualkommunikation: E-Mail und Telefonkonferenz. In: Gert Rickheit (Hg.): Psycholinguistik. Ein internatonales Handbuch. Berlin/New York (Handbuch der Sprach- und Kommunikationswissenschaft, 24), 845-854.

Janich, Nina (2005): Fachkommunikation per E-Mail: Chancen, Risiken, Forschungsperpektiven. In: Mariola Wierzbicka/Matgorzata Sieradzka/Jaromin Homa (Hg.): Moderne deutsche Texte. Frankfurt a.M./Berlin/Bern (Danziger Beiträge zur Germanistik, 16), 197-210.

Kammerer, Sebastian (2013): Information Overload durch E-Mails: Herausforderungen und Lösungsansätze. Hamburg (Schriftenreihe Studien zur Wirtschaftsinformatik 75).

Keller, Reiner (2005): Wissenssoziologische Diskursanalyse als interpretative Analytik. In: Reiner Keller/Andreas Hirseland/Werner Schneider/Willy Vierhöver (Hg.): Die diskursive Konstruktion von Wirklichkeit. Zum Verhältnis von Wissenssoziologie und Diskursforschung. Konstanz, 49-76.

Kleinberger, Ulla (2013): Kontaktuster: Schreiben für Beziehungspflege und Projektkooperation am Beispiel innterbetrieblicher E-Mails. In: Stücheli-Herlach, Peter/Daniel Perrin (Hg.): Schreiben mit System: Texte planen, entwerfen und verbessern für die medienkonvergente PR. Wiesbaden: Springer (Schreiben mit System), 183-190.

Kleinberger, Ulla (2008): Softcommunication - spontane innerbetriebliche Kommunikation ausserhalb vorgegebener Strukturen. In: Florian Menz/Andreas P. Müller (Hg.): Organisationskommunikation. Grundlagen und Analysen der sprachlichen Inszenierung von Organisation. München (Managementkonzepte, 34), 225-242.

Kleinberger Günther, Ulla (2005): „Softcommunication“. Relevanz und Strukturiertheit von spontaner innerbetrieblicher Kommunikation. In: Ulrich Ammon/Klaus J. Mattheier/Peter H. Nelde (Hg.): Wirtschaft und Sprache. Tübingen (Sociolinguistica. Internationales Jahrbuch für Europäische Soziolinguistik, 19), 73-81.

Kleinberger Günther, Ulla (2003): Kommunikation in Betrieben. Wirtschaftslinguistische Aspekte innerbetrieblicher Kommunikation. Bern (Zürcher germanistische Studien, 57).

Kleinberger Günther, Ulla (2002a): Mediennutzung im beruflichen Alltag: Einstellungen, Urteile, Erfahrungen. In: Caja Thimm (Hg.): Unternehmenskommunikation offline/online: Wandelprozesse interner und externer Kommunikation durch neue Medien. Frankfurt a.M./ New York (Bonner Beiträge zur Medienwissenschaft, 1), 36-51.

Kleinberger Günther, Ulla (2002b): Sprachliche Höflichkeit in innerbetrieblichen E-Mails. In: Heinz-Helmut Lüger (Hg.): Höflichkeitsstile. 2. korrigierte Aufl. Bern (Cross Cultural Communication, 7), 147-164.

Kleinberger Günther, Ulla/Carmen Spiegel (2006): Jugendliche schreiben im Internet: grammatische und orthographische Phänomene in normungebundenen Kontexten. In: Christa Dürscheid/Jürgen Spitzmüller (Hg.): Perspektiven der Jugendsprachforschung. Trends and Developments in Youth Language Research. Frankfurt a. M./Berlin/Bern (Sprache, Kommunikation, Kultur. Soziolinguistische Beiträge, 3), 101-116.

Kleinberger Günther, Ulla/Caja Thimm (2000): Soziale Beziehungen und innerbetriebliche Kommunikation: Formen und Funktionen elektronischer Schriftlichkeit in Unternehmen. In: 
Caja Thimm (Hg.): Soziales im Netz. Sprache, Beziehungen und Kommunikationskulturen im Internet. Opladen/Wiesbaden, 262-277.

Kupper, Beda (2008): E-Mail-Archivierung. In: Actualité archivistique suisse, 88-117.

Lamb, Linda/Jerry Peek (1995): What You Need to Nnow Using Email Effectively. Sebastopol, CA.

Lamb, Linda/Martina Wobst/Jerry Peek (1996): Was Sie schon immer wissen wollten: alles über email. Bonn.

Liu, Shuang/Gloria Dall'Alba (2012): Learning intercultural communication through group work oriented to the world beyond the classroom. In: Assessment \& evaluation in higher education 37/1, 19-32.

Luginbühl, Martin (2003): Streiten im Chat. http://www.linguistik-online.de [zit.: 13.1. 2005].

Mandel, Thomas/Gerard Van der Leun (1997): Die zwölf Gebote des Cyberspace. Der kleine NetzKnigge. Mannheim.

McLuhan, Marshall (1995/1962): Die Gutenberg-Galaxis. Das Ende des Buchzeitalters. Bonn.

Meder, Kathrin (2006): E-Mail-Kommunikation: Zwischen Individualität und Konventionen - Eine Untersuchung des Nutzerverhaltens im Alltag. Saarbrücken.

Meier, Gunter (2014): Die E-Mail-Flut bewältigen: E-Mails richtig organisieren - Professionell kommunizieren - Massenaufkommen eindämmen - Kommunikationskultur entwickeln.

5. überarbeitete Aufl. Renningen (Praxiswissen Wirtschaft).

Meier, Stefan/Vivien Sommer (2012): Multimodalität im Netzdiskurs. Methodischmethodologische Betrachtungen zur diskursiven Praxis im Internet. In: Torsten Siever/Peter Schlobinski (Hg.): Entwicklungen im Web 2.0. Ergebnisse des III. Workshops zur linguistischen Internetforschung. Frankfurt a. M. (Sprache, Medien, Innovationen, 3), 97-114.

Merrison, Andrew John/Jack J.Wilson/Bethan L.Davies/Michael Haugh (2012): Getting stuff done. Comparing e-mail requests from students in higher education in Britain and Australia. In: Journal of Pragmatics 44/9, 1077-1098.

Möllering, Martina/Mike Levy (2012): Intercultural competence in computer-mediatedcommunication. An analysis of research methods. In: Melinda Dooly/Robert O’Dowd (Hg.): Researching Online Foreign Language Interaction and Exchange. Theories, Methods and Challenges. Berlin, 233-265.

Mumford, Michael D. (Hg.) (2015): The Psychology of Planning in Organizations: Eesearch and Applications. New York.

Pasfield-Neofitou, Sarah E. (2012a): Online Communication in a Second Language. Social Interaction, Language Use, and Learning Japanese. Bristol (Second language acquisition).

Pasfield-Neofitou, Sarah E. (2012b): "Digital natives" and "native speakers". Competence in computer-mediated communication. In: Farzad Sharifian/Maryam Jamarani (Hg.): Language and Intercultural Communication in the New Era. New York (Routledge studies in language and intercultural communication), 138-159.

Schmidt, Jan (2009): Das neue Netz. Merkmale, Praktiken und Folgen des Web 2.0. Konstanz.

Schmitz, Ulrich (2002a): E-Mails kommen in die Jahre. Telefonbriefe auf dem Weg zu sprachlicher Normalität. In: Arne Ziegler/Christa Dürscheid (Hg.): Kommunikationsform E-Mail. Tübingen (Textsorten, 7), 33-56.

Schmitz, Ulrich (2002b): Briefkommunikation im 20. Jahrhundert. Oldenburg.

Schnitzer, Caroline (2012): Linguistische Aspekte der Kommunikation in den neueren elektronischen Medien. SMS - E-Mail - Facebook. München.

Schwenk, Jörg (2005): Sicherheit und Kryptographie im Internet von sicherer E-Mail bis zu IP-Verschlüsselung. 2., erw. und verb. Aufl. Wiesbaden.

Shipley, David/Will Schwalbe (2008): Erst denken, dann senden!: Die peinlichsten E-Mail-Pannen und wie man sie vermeidet. Dt. Erstausg. München.

Siegert, Paul Ferdinand (2008): Die Geschichte der E-Mail: Erfolg und Krise eines Massenmediums. Bielefeld (Technik - Körper - Gesellschaft, 1). 
Siever, Torsten (2006): Sprachökonomie in den „Neuen Medien“. In: Peter Schlobinski (Hg.): Von *hdl* bis *cul8r*. Sprache und Kommunikation in den Neuen Medien. Mannheim/ Leipzig/Wien/Zürich (Thema Deutsch, 7), 71-88.

Song, Mike/Vicki Halsey/Tim Burress (2008): The Hamster Revolution: How to Manage your Email Before it Manages You. San Francisco.

Spiegel, Carmen/Ulla Kleinberger (2011): Höflichkeitsformen und Höflichkeitsnormen in Internetforen und E-Mails. In: Eva Neuland/Dieter Cherubim (Hg.): Sprachliche Höflichkeit. Der Deutschunterricht 2, 34-43.

Stroinska, Magda/Vikki Cecchetto (2012): Facework in intercultural e-mail communication in the academic environment. In: Farzad Sharifian/Maryam Jamarani (Hg.): Language and Intercultural Communication in the New Era. New York (Routledge studies in language and intercultural communication 1), 160-179.

Thaler, Verena (2012): Sprachliche Höflichkeit in computervermittelter Kommunikation. Tübingen (Linguistik, 70).

Westera, Margo (2007): Generation @. Mögliche Auswirkungen der Nutzung der Kommunikationsplattformen Chat und E-Mail auf das Konfliktverhalten von Jugendlichen zwischen 14 und 16 Jahren. Zürich: Diplomarbeit FH Zürich, Soziale Arbeit.

Wright, David (2013): Stylistic variation within genre conventions in the Enron email corpus: Developing a text-sensitive methodology for authorship research. In: Journal of Speech, Language an the Law 20/1, 45-75.

Ziegler, Arne/Christa Dürscheid (Hg.) (2002): Kommunikationsform E-Mail. Tübingen (Textsorten, 7).

\section{Primärquellen}

Libess_1: http://www.libess.de/internetstatistiken-2012/ [zit 29.12. 2013]

Netcare_1: http://www.pharmasuisse.org/DE/dienstleistungen/themen/seiten/netcare.aspx [zit. 1.1. 2014]

Radicati Group: http://www.radicati.com [zit 29.12. 2013]

Statista_1: http://de.statista.com/statistik/daten/studie/247225/umfrage/schaetzung-zur-zahlder-taeglich-verschickten-e-mails/ [zit. 29.12. 2013]

FAZ_Höfinghoff: Höfinghoff, Tim (2008): E-Mails blockieren den Kopf. http://www.faz.net/aktuell/ beruf-chance/arbeitswelt/arbeitsalltag-e-mails-blockieren-den-kopf-1514487.html [zit. 31. 12. 2013].

PGP: http://datatracker.ietf.org/wg/openpgp/charter/ [zit 6.1. 2014].

GnuPG: http://gnupg.org/ [zit 6.1.2014].

TKG_Dt: Telekommunikationsgesetz § 110: Telekommunikationsgesetz § 110 „Umsetzung von Überwachungsmaßnahmen, Erteilung von Auskünften“, http://www.gesetze-im-internet.de/ tkg_2004/_110.html, Bundesministerium der Justiz, (nichtamtliches Verzeichnis). [zit 6.1.2014].

TKG_CH: http://www.admin.ch/opc/de/classified-compilation/20002506/index.html [zit 6.1.2014].

TKG_AT = http://www.ris.bka.gv.at/Dokumente/RegV/REGV_COO_2026_100_2_654439/REGV_ COO_2026_100_2_654439.pdf [zit 6.1. 2014]. 